\title{
Síndrome intestino irritable
}

\author{
Irritable bowel syndrome
}

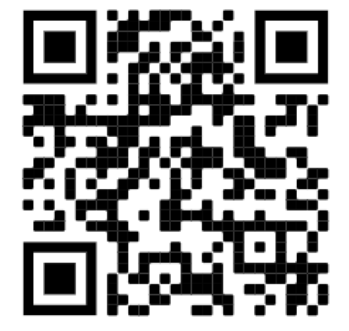

Recibido 25/08/2020

\author{
${ }^{1}$ Dra. Nathalya Zeledón Corrales \\ Área de salud de Oreamuno, Cartago, Costa Rica \\ iD https://orcid.org/0000-0001-9540-1840 \\ ${ }^{2}$ Dr. José Antonio Serrano Suárez \\ Investigador independiente, San José, Costa Rica \\ (D) https://orcid.org/0000-0003-0304-1986 \\ ${ }^{3}$ Dr. Sebastián Fernández Agudelo \\ Investigador independiente, San José, Costa Rica \\ (D) https://orcid.org/0000-0002-3871-6827
}

Corregido $16 / 09 / 2020$
Aceptado

05/12/2020

\section{RESUMEN}

El síndrome de intestino irritable es una patología frecuente en la práctica clínica. Está caracterizado por dolor abdominal, alteración en el hábito intestinal, distensión abdominal y flatulencias. Los criterios Roma IV son una herramienta para ayudar al clínico a definir el diagnóstico y la necesidad de realizar pruebas en el paciente. No hay una causa especifica identificada, es conocido que existe una alteración en el eje bidireccional cerebro-intestino el cual está influenciado por múltiples factores. Existen 4 subtipos, síndrome de intestino irritable con constipación, con diarrea, mixto e inespecífico. De acuerdo con el subtipo se designa el mejor tratamiento. Existen terapias farmacológicas y no farmacológicas que permiten brindar al paciente el enfoque multidisciplinario que requiere esta patología debido a su heterogénesis.

PALABRAS CLAVE: síndrome intestino irritable; colitis; enfermedades del sistema digestivo; diarrea; estreñimiento.
Irritable bowel syndrome is a frequent pathology in clinical practice. It is characterized by abdominal pain, alteration in bowel habit, bloating and flatulence. The Rome IV criteria are a tool to help the clinician define the diagnosis and the need to carry out tests on the patient. There is no specific cause identified, it is known that there is an alteration in the bidirectional brain-intestine axis which is influenced by multiple factors. There are 4 subtypes, irritable bowel syndrome with constipation, with diarrhea, mixed and nonspecific. According to the subtype, the best treatment is designated. There are pharmacological and non-pharmacological therapies that allow providing the patient with the multidisciplinary approach

\footnotetext{
${ }^{1}$ Médica general, graduada de la Universidad de Ciencias Médicas (UCIMED), cod. MED15226 Correo: nathaliazeledon30@gmail.com

${ }^{2}$ Médico general, graduado de la Universidad de Ciencias Médicas (UCIMED), cod. MED15665. Correo: jose serrano07@hotmail.com

${ }^{3}$ Médico general con Maestria universitario en Fisiología de la Universidad de Valencia, graduado de la Universidad de Ciencias Médicas (UCIMED), cod. MED15221.Correo: sebastian.fernandez.cr.1992@gmail.com
} 
that this pathology requires due to its heterogenesis.

KEYWORDS: irritable bowel syndrome; colitis; digestive system diseases; diarrhea; constipation.

\section{INTRODUCCIÓN}

El síndrome del intestino irritable (SII) es un trastorno gastrointestinal funcional común. Esta afección crónica es caracterizada por dolor abdominal, alteración en el hábito intestinal, distensión abdominal e hinchazón (1).

Los criterios de Roma IV, fueron establecidos por un grupo de expertos en trastornos gastrointestinales funcionales y constituyen el estándar actual para diagnosticar el SIl. Según estos criterios el SII se clasifica en cuatro subtipos: SII con diarrea, SII con estreñimiento, SII con síntomas mixtos de estreñimiento y diarrea, o SII inespecífico (2).

Con una prevalencia del $10 \%$ al $20 \%$ el SII tiene un impacto negativo en la calidad de vida de las personas y productividad laboral, como consecuencia los pacientes sufren disminución en sus ingresos, dificultad a la hora de socializar y de viajar.

Además, representa una carga socioeconómica importante porque los pacientes afectados utilizan más recursos sanitarios $(1,3)$.

El SIl es una condición heterogénea compleja con patogenia multifactorial por lo que no existe un tratamiento que sea beneficioso para todos los pacientes. El tratamiento debe ser individualizado basado en el subtipo y gravedad de cada paciente (2).

El objetivo de este trabajo es revisar la bibliografía más actualizada para comprender mejor esta patología y así tomar mejores decisiones en cuanto a manejo y terapia para estos pacientes.

\section{MÉTODO}

Para esta revisión bibliográfica la búsqueda de información se realizó en la base de datos PubMed y Scielo. Se utilizaron los siguientes descriptores: Síndrome de intestino irritable, Colitis, Distensión abdominal. Fueron seleccionados artículos científicos de revisión y metaanálisis publicados entre el 2016 y el 2020. Para la selección de las publicaciones se analizó el texto completo y se identificaron las más relevantes para desarrollar el artículo.

\section{EPIDEMIOLOGÍA}

La prevalencia mundial del SII es del $10 \%$ al $20 \%$. Es el trastorno gastrointestinal más comúnmente diagnosticado. El SII a menudo se manifiesta en la infancia, aunque la prevalencia máxima parece ser en la edad adulta temprana. Las mujeres se ven afectadas en una proporción de 2: 1 con respecto a los hombres y hasta la mitad de las personas afectados buscan atención médica (4).

En 2018 se realizó un estudio liderado por expertos en el campo del SIl y financiado por la industria farmacéutica donde se destacó la importancia de considerar el efecto del SII en todo el mundo. El estudio de reveló que a las personas con SII les toma en promedio 4 años recibir un diagnostico con la sensación de que su médico no las toma en serio. Además, el estudio mostró que las personas pueden sentirse estigmatizadas por su diagnóstico y pueden experimentar problemas para acceder a un tratamiento y apoyo efectivo (3). 


\section{FISIOPATOLOGÍA}

La fisiopatología del SIl es compleja y multifactorial. Todavía no se ha determinado una causa especifica. Numerosos estudios han desarrollado hipótesis sobre la fisiopatología del SII. Existen varios factores o mecanismos involucrados en la patogénesis del SII capaces de ejercer influencia en el eje bidireccional cerebrointestino así como en el intestino de manera local (4). Dentro de estos se pueden resaltar:

- Genética: muchos pacientes con SII refieren tener familiares con el mismo diagnóstico, los estudios han analizado la agregación familiar del SII y sugieren un componente genético subyacente. Las investigaciones se han centrado en tratar de identificar posibles marcadores genéticos en el SII. Se ha sugerido que un polimorfismo genético en el promotor de la región del gen SLC6A4 que codifica el transportador de recaptación de serotonina (SERT) podría estar asociada con el SIl. Otros estudios han identificado variantes patogénicas raras en genes que codifican sacarasaisomaltasa, un canal de sodio dependiente de voltaje, lo que sugiere que los síntomas del SII en una pequeña proporción de pacientes podrían estar relacionados con intolerancia a los disacáridos o canalopatías iónicas. A pesar de estos hallazgos todavía falta esclarecer si realmente contribuyen a los mecanismos fisiopatológicos (3).

- Sistema inmunitario y trastornos de la barrera epitelial: los pacientes que sufren de SII suelen tener un aumento en varias células proinflamatorias como los mastocitos y los linfocitos, así como un aumento en ciertas citocinas inflamatorias como el factor de necrosis tumoral alfa, la interleucina 6, el factor de inducción linfocitario, el factor de crecimiento nervioso y la interleucina 1 . La inflamación provocada por estos factores causa un aumento en la permeabilidad de la barrera con una consecuente pérdida de fluido intersticial hacia la luz intestinal que puede explicar la aparición súbita de diarrea y el estreñimiento al normalizarse los niveles (4).

- Trastornos en la motilidad y sensibilidad visceral: en los pacientes con SII se ha demostrado que hay un aumento de retención de gas acompañado de una disminución en la velocidad del tránsito intestinal desde el duodeno hasta el recto. Este aumento de los niveles de gas acompañado del tiempo de tránsito intestinal causa un aumento en la sensación de distensión abdominal y dolor visceral en sujetos con SII (4).

- Alimentación: los pacientes con frecuencia refieren que ciertos alimentos desencadenan sus síntomas abdominales. Algunos pacientes con SII han reportado un beneficio sintomático al reducir las cantidades de oligosacáridos fermentables, disacáridos, monosacáridos y polioles (FODMAP) en su dieta. Estas moléculas tienen un efecto osmótico, lo que aumenta la carga de líquido en el tracto gastrointestinal y aumenta la distensión intestinal. También conducen a la fermentación bacteriana con una mayor producción de gas en el tracto intestinal (5).

- Microbioma y disbiosis gastrointestinal: investigaciones han 
evidenciado que una interrupción en la biodiversidad, riqueza y composición del microbiota intestinal (proceso llamado disbiosis), juega una función clave en la patogénesis del SII. La disbiosis puede ocurrir a través de varios mecanismos como crecimiento excesivo o la desaparición de especies bacterianas específicas, alteraciones en la riqueza de bacterias y mutación o transferencia de genes. En pacientes con SII, la disbiosis gastrointestinal se ha asociado con un aumento de la percepción visceral del dolor y una mayor permeabilidad de la mucosa provocada por defectos en la barrera epitelial mucosa, que interfiere con la homeostasis inmune intestinal y posteriormente promueve la inflamación intestinal y aumenta la activación inmunitaria celular y mucosa. También se ha asociado con cambios en la motilidad intestinal y alteraciones del sistema nervioso entérico. Existe también un eje microbiota- intestinocerebro ya que se demostró que la microbiota intestinal puede verse afectada en cuanto a motilidad intestinal, secreción y función inmune, por la actividad cerebral (6).

- Factores

psicológicos:

tradicionalmente, el SII se ha visto como un trastorno del cerebro-intestino debido a su alta asociación con condiciones psiquiátricas y psicológicas coexistentes. El estrés, la ansiedad o la depresión se asocia con frecuencia al SII y a la exacerbación de síntomas. La interacción entre el sistema nervioso central y el sistema entérico es importante en la fisiopatología del SII y funciona de manera bidireccional. El sistema nervioso central puede alterar la fisiología intestinal, como la motilidad o la sensibilidad visceral, que, a su vez media en la sintomatología del SII, como el tránsito y el hábito intestinal o la percepción del dolor. Del mismo modo, los cambios en el intestino pueden dar retroalimentación al cerebro, lo que resulta en efectos sobre el bienestar psicológico y la salud. Es importante tener en cuenta que los síntomas psicológicos pueden haberse desarrollado como consecuencia de la gravedad y el efecto del SIl en el paciente, o podrían haber estado presentes antes del inicio de los síntomas gastrointestinales $(1,3)$.

- Sexo femenino: hay una mayor prevalencia de SII en pacientes femeninas,

Las variaciones biológicas en las hormonas sexuales, los mecanismos serotoninérgicos, las vías del dolor, los problemas psicosociales y la comorbilidad uroginecológica dan como resultado diferencias entre pacientes femeninos y masculinos. Las mujeres presentan mayor percepción del dolor, esto se ve manifestado en los efectos de las hormonas sexuales en la sensación del dolor, una mayor activación de los centros asociados con las emociones cerebrales durante la estimulación visceral, el estrés, el dolor en los primeros años de la vida y diferencias en los mecanismos serotoninérgicos (7).

\section{CLÍNICA}

Los síntomas característicos del SIl pueden ser variados dependiendo de la vía fisiopatológica que sea dominante en cada paciente. El dolor abdominal es a menudo el principal síntoma de esta enfermedad y usualmente se asocia a la defecación,

e645 Revista Médica Sinergia Vol.6 (2), Febrero 2021 - ISSN:2215-4523 / e-ISSN:2215-5279 
aunque es normal que esté presente también durante períodos de estreñimiento. La distensión abdominal también es un síntoma común y está asociado a un aumento en la cantidad de gas intestinal que puede llevar a un aumento del meteorismo. El SIl tiene un componente interesante ya que puede variar el patrón defecatorio de los sujetos y producir tanto diarrea como estreñimiento. Este comportamiento puede ser predominantemente de uno de estos dos tipos o puede tener un componente mixto. La frecuencia y la forma de las deposiciones está directamente relacionado al subtipo de SIl que se padezca (2-4). En la FIGURA 1 se puede apreciar la escala de Bristol que es una herramienta útil para poder medir objetivamente el patrón de heces y así poder determinar si predomina más la diarrea o el estreñimiento.

\section{DIAGNÓSTICO}

El diagnóstico de SIl comienza con una historia clínica detallada. Aparte de evaluar la clínica detallada anteriormente es importante tomar en cuenta la cronicidad de los síntomas (2).

Manning y colaboradores fueron los primeros en proponer un método para diagnosticar el SII basado en la presencia de ciertos síntomas gastrointestinales reportados por el paciente. Esto condujo al desarrollo de los criterios de Roma que, en los últimos 20 años, se han convertido en el estándar de oro para el diagnóstico de SIl. La versión más reciente, Roma IV, se publicó en 2016 (8). En la TABLA 1 se citan los criterios de Manning y los criterios de Roma IV.

Los criterios deben cumplirse durante los últimos tres meses y los síntomas haber comenzado un mínimo de seis meses antes del diagnóstico (9). Los estudios muestran que la sensibilidad y la especificidad de los criterios de Roma oscilan entre $69 \%-96 \%$ y $72 \%-85 \%$, respectivamente (2).

Según los criterios Roma IV, el SIl se clasifica en cuatro subtipos: SII con diarrea, SII con estreñimiento, SII con síntomas mixtos de estreñimiento y diarrea, o SII no especificado. En la TABLA 2 se citan los criterios para los subtipos de SII. Los criterios Roma IV para subtipos de SII se basan en la percepción del paciente de su tipo predominante de consistencia de heces utilizando la escala de Bristol. Determinar el patrón de síntomas predominantes de un paciente es útil para guiar el manejo ya que los subgrupos responden de manera diferente a las diversas intervenciones terapéuticas. Se debe recalcar que los patrones de síntomas individuales pueden cambiar con el tiempo (10).

Diferentes guías y estudios sobre el manejo del SIl establecen que, si un paciente presenta síntomas que cumplen con los criterios Roma IV y no presenta signos de alarma, el médico puede hacer un diagnóstico positivo de SII sin recurrir a exámenes de gabinete. Solicitar estudios de laboratorio o morfológicos de forma rutinaria no está respaldado por la evidencia científica, a excepción de que el médico observe signos de alarma o sospecha clínica de patología orgánica o metabólica $(1,9)$.

Si el médico tiene la sospecha de que existe otra patología puede realizar más estudios con el objetivo de descartar patología orgánica. Se recomienda solicitar exámenes sencillos primero como un examen de sangre, pruebas de función metabólica. Estas pruebas deben ir dirigidas en orden de aclarar una sospecha clínica especifica como por ejemplo si el paciente además presenta síntomas relacionados con hipertiroidismo o hipotiroidismo se deben solicitar pruebas de función tiroidea o si el 
FIGURA 1. Escala de heces de Bristol

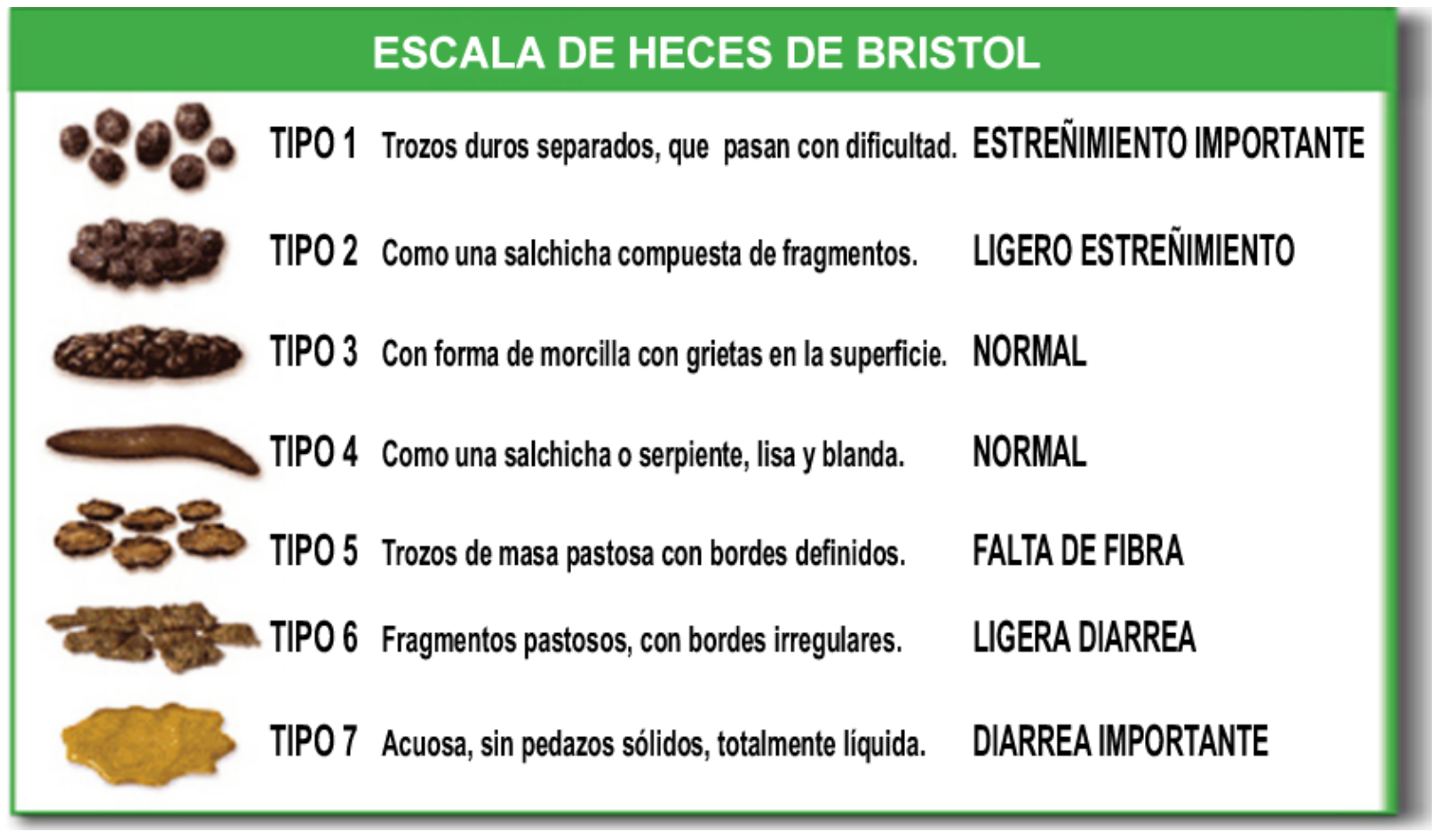

Fuente: . "File:Escala de Bristol.1.png" by Cabot Health, Bristol Stool Chart is licensed with CC BY-SA 3.0.

To view a copy of this license, visit https://creativecommons.org/licenses/by-sa/3.0

TABLA 1. Criterios diagnósticos del síndrome de intestino irritante

\begin{tabular}{|c|c|}
\hline Criterios de Manning & Criterios de Roma IV \\
\hline $\begin{array}{l}\text { Para establecer el diagnóstico de Sll debe de haber } \\
3 \text { o más criterios positivos: } \\
\text { 1. Alivio del dolor con la defecación } \\
\text { 2. Heces pastosas al inicio del dolor } \\
\text { 3. Inicio del dolor asociado a un aumento de } \\
\text { los movimientos intestinales } \\
\text { 4. Distensión abdominal visible } \\
\text { 5. Diarrea con moco } \\
\text { 6. Sensación de evacuación incompleta }\end{array}$ & $\begin{array}{l}\text { Para establecer el diagnóstico de Sll debe de haber } \\
\text { dolor abdominal recurrente por más de un día a la } \\
\text { semana por } 3 \text { meses o más durante los últimos } 6 \\
\text { meses previos al diagnóstico asociado a dos o más } \\
\text { criterios positivos: } \\
\text { 1. Dolor asociado a la defecación } \\
\text { 2. Cambios en la frecuencia de las heces } \\
\text { 3. Cambios en la apariencia de las heces }\end{array}$ \\
\hline
\end{tabular}


TABLA 2. Criterios diagnósticos de los suptipos de síndrome de intestino irritante

\begin{tabular}{|l|l|}
\hline \multicolumn{1}{|c|}{ Subtipo } & \multicolumn{1}{|c|}{ Criterio de Roma IV } \\
\hline SII-C (SII con predominio de estreñimiento) & $\begin{array}{l}>25 \% \text { de las heces catalogadas con la Escala de } \\
\text { Bristol 1-2 y }<25 \% \text { de las heces catalogadas con la } \\
\text { Escala de Bristol 6-7 }\end{array}$ \\
\hline SII-D (SII con predominio de diarrea) & $\begin{array}{l}>25 \% \text { de las heces catalogadas con la Escala de } \\
\text { Bristol 6-7 y }<25 \% \text { de las heces catalogadas con la } \\
\text { Escala de Bristol 1-2 }\end{array}$ \\
\hline SII-M (SII con hábitos intestinales mixtos) & $\begin{array}{l}>25 \% \text { de las heces catalogadas con la Escala de } \\
\text { Bristol 1-2 y }>25 \% \text { de las heces catalogadas con la } \\
\text { Escala de Bristol 6-7 }\end{array}$ \\
\hline SII-U (no clasificado) & $\begin{array}{l}\text { Los paciente cumples con los criterios para el } \\
\text { diagnóstico de SII pero su patrón de heces no se } \\
\text { puede categorizar en ninguno de los otros subtipos }\end{array}$ \\
\hline Abreviatura: SII: síndrome de intestino irritante & \\
\hline $\begin{array}{l}\text { Fuente: traducido y adaptado de Grad S, Dumitrascu DL. Irritable Bowel Syndrome Subtypes: New Names for Old Medical } \\
\text { Conditions. Dig Dis. 2020;38(2):122-7 }\end{array}$
\end{tabular}

paciente recientemente recibió antibióticos es importante descartar una infección por Clostridium difficile por lo que se debe realizar un análisis de heces $(2,8)$.

Es importante tener en cuenta la medición de biomarcadores para el diagnóstico del SIl. Un metaanálisis desarrollado en el 2015 por Menees et al. Donde se compararon diferentes marcadores séricos y fecales en adultos con SII revelo las siguientes conclusiones:

- Primero, la proteína $\mathrm{C}$ reactiva demostró tener una alta probabilidad predictiva para el SII de hasta $45,7 \%$ a los $1,3 \mathrm{mg} / \mathrm{dl}$ en sangre. Pero esta bajó considerablemente a menos de $10 \%$ a los $2,7 \mathrm{mg} / \mathrm{dl}$.

- Segundo, la velocidad de eritrosedimendación (VES) tuvo un valor predictivo de $6,9 \%$ a $10 \mathrm{~mm} / \mathrm{h}$.

- Tercero, la calprotectina fecal tiene un valor predictivo de $11,6 \%$ a $20 \mu \mathrm{g} / \mathrm{g}$ de heces y de $7,6 \%$ a los $1000 \mu \mathrm{g} / \mathrm{g}$ con una probabilidad pico de $18,8 \%$ a los
$280 \mu \mathrm{g} / \mathrm{g}$. Se vio que valores mayores a $100 \mu \mathrm{g} / \mathrm{g}$ de asocian a $>15 \%$ de probabilidad de SII. Se determinó que esta prueba tiene un alto valor predictivo negativo pero un muy bajo valor predictivo positivo por lo que es capaz de excluir el SII.

- Cuarto, la lactoferrina fecal tiene $74 \%$ de valor predictivo positivo a los $2960 \mu \mathrm{g} / \mathrm{g}$ de heces, pero es incapaz de diferenciar entre SII y enfermedad inflamatoria intestinal por lo que su utilidad diagnóstica es limitada (11).

Ninguno de los marcadores séricos o fecales es capaz de identificar con precisión el SII, pero la proteína $\mathrm{C}$ reactiva y la calprotectina fecal sirven para descartar enfermedad inflamatoria intestinal en pacientes con síntomas de SII. Es razonable que el médico tratante ordene niveles de proteína $\mathrm{C}$ reactiva y calprotectina fecal como un examen complementario para aumentar la seguridad en el diagnóstico de SII, sin embargo, no es recomendable utilizarlo 
como única herramienta diagnostica ya que no tiene suficiente valor predictivo positivo $y$ solo sirven para excluir diagnósticos diferenciales. La lactoferrina y la VES no demostraron ser de utilidad en esta patología $(2,8,11)$.

Con la evidencia disponible, no se recomienda la realización de colonoscopía en individuos jóvenes con sospecha de SII, sin signos de alarma, esta debe reservarse para personas mayores o que presenten exámenes de gabinete alterados o signos de alarma (8).

No se ha demostrado que la realización de radiología simple de abdomen o de enema opaco tenga utilidad para encontrar características morfológicas que ayuden a realizar el diagnóstico (9).

\section{SIGNOS DE ALARMA}

Es importante también tomar en cuenta los signos de alarma a la hora de hacer el diagnóstico de esta patología ya que estos nos ayudan a identificar otros diagnósticos diferenciales del SIl que pueden potencialmente ser mucho más graves (2). Los signos de alarma incluyen pérdida de peso, despertar nocturno debido a síntomas gastrointestinales, sangre en las heces, antecedentes familiares de cáncer de colon o enfermedad inflamatoria intestinal, uso reciente de antibióticos y fiebre $(2,8)$.

\section{DIAGNÓSTICOS DIFERENCIALES}

Entre los diagnósticos diferenciales para el SIl podemos destacar las patología tiroideas, la infección por Clostridium difficile, enfermedad celiaca, enfermedad inflamatoria intestinal, diverticulitis, la colitis microscópica y las neoplasias intestinales principalmente el cáncer de colon y recto $(2,4,5)$.

\section{TRATAMIENTO}

El tratamiento del síndrome de intestino irritable requiere un enfoque multidisciplinario debido a su heterogénesis. Se debe iniciar identificando el subtipo de SII y la gravedad de los síntomas que presenta el paciente para así determinar el mejor tratamiento. Los pacientes deben estar tranquilos sobre el curso benigno del SII, es muy importante explicar las distintas terapias al paciente para lograr un correcto apego al tratamiento. Existen medidas farmacológicas y no farmacológicas que han demostrado mejorar considerablemente los síntomas $(1,2,12)$.

\section{No farmacológicos}

- Dieta: inicialmente se recomienda realizar cambios en la dieta. Se debe dar educación nutricional al paciente sobre alimentos que podrían desencadenar síntomas como distención y dolor abdominal. Se ha demostrado que la dieta baja en oligosacáridos fermentables, disacáridos, monosacáridos y polioles (FODMAP) reduce los síntomas en pacientes con SII. La disminución de los alimentos fermentables reduce la distensión gaseosa, alterando así la respuesta al dolor (12). El paciente debe evitar comidas grasosas, legumbres, repollo, coliflor, brócoli, algunos carbohidratos, exceso de cafeína y bebidas carbonatadas. Investigaciones científicas han demostrado que algunos pacientes muestran mejoría de síntomas al llevar una dieta libre de gluten, sin embargo, no todos presentan este beneficio. Es muy importante que los cambios en la dieta se realicen bajo supervisión nutricional $(1,2)$. Aunque el 
incremento de fibra en la dieta se ha recomendado durante muchos años se ha visto que las fibras insolubles pueden exacerbar algunos síntomas principalmente en pacientes con SII-C. Sin embargo, existen reportes de pacientes se han visto beneficiados al aumentar la fibra en la dieta $(1,12)$.

- Atención psicológica: es importante brindar educación sobre estrés psicosocial y crear estrategias para que los pacientes puedan manejar el estrés. El ejercicio moderado, prácticas como yoga y la meditación pueden ayudar a disminuir el estrés.

Es importante buscar otras causas de estrés como trastornos del sueño ya que al corregirlos se ha visto mejoría (2). Se realizó un estudio para determinar cuál tratamiento no farmacológico era más efectiva para el SII, dentro de las terapias que se analizaron se encuentran el manejo del estrés, terapia cognitivo conductual, terapia de relajación, hipnoterapia y meditación.

No se logró identificar cuales tienen mejores resultados, pero se observó una mejoría considerable en los pacientes con cualquier subtipo de SII que realizaron este tipo de terapia. Se ha visto que a largo plazo la hipnoterapia es la que demostró mejores resultados (13).

\section{Farmacológicos:}

- Antiespasmódicos: son utilizados independientemente del subtipo de SII, representan la primera línea para aliviar dolor abdominal tipo cólico y espasmos. Actúan relajando el musculo liso y causan alivio por periodos cortos de tiempo. Sin embargo, su uso tiene que ser limitado en el subtipo de SII con constipación ya que como efecto secundario podría empeorar el estreñimiento $(12,14)$.

- Antidiarreicos: están indicados en el SII-D. La Loperamida actúa prolongando el tránsito intestinal. Dicho medicamento mejora la consistencia de las heces; sin embargo, no causa alivio en el dolor abdominal. Se debe de tomar en cuenta que su uso continuo no ha demostrado una mejoría de síntomas.

La Asociación Canadiense de Gastroenterología (CAG según sus siglas en inglés) no lo aconseja teniendo en cuenta que es una recomendación condicional por su evidencia de muy baja calidad.

Otro medicamento utilizado es el eluxodoline, un agonista de los receptores opioides $\mu$ y $\mathrm{k}$ y antagonista del receptor opioide $\delta$, que actúa mejorando la consistencia de las heces y reduce el dolor abdominal en algunos pacientes. Dentro de los efectos secundarios presenta nauseas, estreñimiento y dolor abdominal. La CAG lo recomienda para tratar el SIl con diarrea, pero solo como tratamiento sintomático y no como tratamiento de uso diario $(5,15,16)$.

- Antagonistas del receptor 5hidroxitriptamina (5-HT) tipo 3: como el Alosetrón y Ondasetron los cuales tienen un efecto sobre las neuronas entéricas, periféricas y centrales que afectan la regulación del dolor visceral, las secreciones gastrointestinales y el tránsito colónico, los cuales se relacionan con la fisiopatología del SII. Actualmente está indicado solo en mujeres con SII-D grave (13). 
- Secuestradores de sales biliares: como colestiramina que se une a los ácidos biliares lo que puede aumentar el tiempo de tránsito de las heces (4).

- Laxantes: estos medicamentos son utilizado para los pacientes que presentan SII con constipación. Este grupo de medicamentos tiene un efecto positivo en reducir la constipación, pero no tiene efectos sobre el dolor abdominal. Dentro de los más comúnmente utilizados se puede mencionar lactulosa, leche magnesia y polietilenglicol (PEG). El PEG es de los más utilizados ya que presenta menos efectos adversos y proporciona una reducción del discomfort abdominal. Si los síntomas de estreñimiento persisten o si el paciente no puede tolerar el PEG, se puede adicionar lubiprostone 0 linaclotida. El lubiprostone es un activador de los canales de cloro que suavizan las heces y facilita la evacuación de estas, también ha demostrado ser útil en el manejo del SII $(2,5,12)$.

- Antidepresivos: los antidepresivos tienen propiedades analgésicas además de mejorar del estado de ánimo. Los antidepresivos tricíclicos (TCAS según sus siglas en inglés) reducen el tiempo de tránsito intestinal por sus efectos anticolinérgicos, lo que favorece su uso en el SII con diarrea. Un metaanálisis realizado en el 2015 concluyó que los antidepresivos tricíclicos en adultos fueron significativamente más efectivos que el placebo para mejorar los síntomas globales del SIl (14). También se ha demostrado que los inhibidores selectivos de la recaptación de serotonina (ISRR) mejoran los síntomas con respecto al placebo, mientras que los inhibidores de la recaptación de serotonina y norepinefrina tienen poca evidencia para respaldar su uso (12).

- Probióticos: los probióticos son utilizados para lograr una homeostasis en la microbiota intestinal. Dentro de los efectos adversos presenta un aumento en la producción de gas, causando meteorismo y distención abdominal. Se realizó un metaanálisis donde se observó que el uso de un solo probiótico por 8 semanas es beneficial para los pacientes. Sin embargo, concluyó que se necesita más investigación científica para recomendarlo como tratamiento $(4,16,17)$.

- Canabinoides: dentro de las nuevas alternativas de tratamiento se ha utilizado canabidiol (CBD) que se podría recomendar para SII ya que se ha visto una disminución en dolor abdominal y espasmos, sin embargo se necesita más investigación para considerarlo como tratamiento $(4,18)$.

En la TABLA 3 se resumen los principales tratamientos específicos para los subtipos SII-C y SII-D.

\section{CONCLUSIÓN}

El SIl es una condición prevalente y multifactorial con un alto impacto socioeconómico que afecta de manera significativa la calidad de vida de los pacientes. Si el paciente cumple con los criterios Roma IV, no presenta signos de alarma y el médico no sospecha de patología orgánica se puede realizar el diagnóstico de SIl sin necesidad de enviar exámenes de laboratorio. 
TABLA3. Principales tratamientos para los subtipos de síndrome de intestino irritable

\begin{tabular}{|c|c|}
\hline Subtipo & Terapias farmacológicas \\
\hline SII-C (SII con predominio de estreñimiento) & $\begin{array}{l}\text { - } \text { Fibras solubles como el lubiprostone y el PEG } \\
\text { 3350. } \\
\text { - } \quad \text { Fibras insolubles. } \\
\text { - }\end{array}$ \\
\hline SII-D (SII con predominio de diarrea) & $\begin{array}{l}\text { - } \text { Loperamida. } \\
\text { - } \\
\text { - Antiedoline. } \\
\text { diciclomina. } \\
\text { - ISRR y TCAS como la fluoxetina y las } \\
\text { amitriptilina. Secuestradores de sales biliares } \\
\text { como la colestiramina. } \\
\text { - Antagonistas del receptor 5HT3 como el } \\
\text { alosetron }\end{array}$ \\
\hline
\end{tabular}

Abreviatura: SII: síndrome de intestino irritante. TCAS: antidepresivos tricíclicos. ISRR: inhibidores selectivos de la recaptación de serotonina

Fuente: adaptada de Defrees DN, Bailey J. Irritable Bowel Syndrome: Epidemiology, Pathophysiology, Diagnosis, and Treatment. Prim Care Clin Off Pract. 1 de diciembre de 2017;44(4):655-71.

El tratamiento de cada paciente debe ser individualizado de acuerdo con el subtipo de SII que presente. Además, la terapia debe ser multidisciplinaria y orientada con respecto a los factores de riesgo de cada paciente. Las terapias no farmacológicas como cambios en la dieta, manejo del estrés, terapia cognitivo conductual, terapia de relajación, hipnoterapia y meditación han demostrado ser efectivas en los pacientes.
Existen múltiples factores o mecanismos capaces de ejercer influencia en el eje bidireccional cerebro-intestino los cuales no son comprendidos completamente y algunos todavía no han sido estudiados por lo que brindan una ventana de investigación para desarrollar mejores terapias y dar mejor calidad de vida a los pacientes de difícil manejo.

\section{REFERENCIAS}

1. Ford AC, Lacy BE, Talley NJ. Irritable Bowel Syndrome. N Engl J Med. 29 de 2017;376(26):256678.htps://doi.org/10.1056/NEJMra1607547

2. Sultan S, Malhotra A. Irritable Bowel Syndrome. Ann Intern Med. 6 de junio de 2017;166(11):ITC8-96. https://doi.org/10.7326/AITC201706060

3. Black CJ, Ford AC. Global burden of irritable bowel syndrome: trends, predictions and risk factors. Nat Rev Gastroenterol Hepatol. agosto de 2020;17(8):473-86. https://doi.org/10.1038/s41575-020-0286-8

4. Defrees DN, Bailey J. Irritable Bowel Syndrome: Epidemiology, Pathophysiology, Diagnosis, and Treatment. Prim Care Clin Off Pract. 1 de diciembre de 2017;44(4):655-71. https://doi.org/10.1016/j.pop.2017.07.009 
5. Grad S, Dumitrascu DL. Irritable Bowel Syndrome Subtypes: New Names for Old Medical Conditions. Dig Dis. 2020;38(2):122-7. https://doi.org/10.1159/000505287

6. Mearin F, Ciriza C, Mínguez M, Rey E, Mascort JJ, Peña E, et al. Guía de práctica clínica: síndrome del intestino irritable con estreñimiento y estreñimiento funcional en adultos: concepto, diagnóstico y continuidad asistencial. (Parte 1 de 2). Aten Primaria. 1 de enero de 2017;49(1):42-55. https://doi.org/10.1016/j.aprim.2016.11.003

7. Mari A, Abu Baker F, Mahamid M, Sbeit W, Khoury T. The Evolving Role of Gut Microbiota in the Management of Irritable Bowel Syndrome: An Overview of the Current Knowledge. J Clin Med. marzo de 2020;9(3):685.https://doi.org/10.3390/jcm9030685

8. Moayyedi P, Simrén M, Bercik P. Evidence-based and mechanistic insights into exclusion diets for IBS. Nat Rev Gastroenterol Hepatol. julio de 2020;17(7):406-13. https://doi.org/10.1038/s41575-020-0270-3

9. Camilleri M. Sex as a biological variable in irritable bowel syndrome. Neurogastroenterol Motil. 2020;32(7):e13802. https://doi.org/10.1111/nmo.13802

10. Black CJ, Thakur ER, Houghton LA, Quigley EMM, Moayyedi P, Ford AC. Efficacy of psychological therapies for irritable bowel syndrome: systematic review and network meta-analysis. Gut. 1 de agosto de 2020;69(8):144151. https://doi.org/10.1136/gutjinl-2020-321191

11. Menees SB, Powell C, Kurlander J, Goel A, Chey WD. A meta-analysis of the utility of C-reactive protein, erythrocyte sedimentation rate, fecal calprotectin, and fecal lactoferrin to exclude inflammatory bowel disease in adults with IBS. Am J Gastroenterol. marzo de 2015;110(3):444-54. https://doi.org/10.1038/ajg.2015.6

12. Black CJ, Ford AC. Rational investigations in irritable bowel syndrome. Frontline Gastroenterol. 1 de marzo de 2020;11(2):140-7. https://doi.org/10.1136/flgastro-2019-101211

13. Rawla P, Sunkara T, Raj JP. Updated review of current pharmacological and non-pharmacological management of irritable bowel syndrome. Life Sci. 1 de noviembre de 2018;212:176-81. https://doi.org/10.1016/j.lfs.2018.10.001

14. Moayyedi P, Andrews CN, MacQueen G, Korownyk C, Marsiglio M, Graff L, et al. Canadian Association of Gastroenterology Clinical Practice Guideline for the Management of Irritable Bowel Syndrome (IBS). J Can Assoc Gastroenterol. abril de 2019;2(1):6-29. https://doi.org/10.1093/jcag/gwy071

15. Brenner DM, Sayuk GS. Current US Food and Drug Administration-Approved Pharmacologic Therapies for the Treatment of Irritable Bowel Syndrome with Diarrhea. Adv Ther. 1 de enero de 2020;37(1):83-96. https://doi.org/10.1007/s12325-019-01116-z

16. Li B, Liang L, Deng H, Guo J, Shu H, Zhang L. Efficacy and Safety of Probiotics in Irritable Bowel Syndrome: A Systematic Review and Meta-Analysis. Front Pharmacol. 2020;11:332.https://doi.org/10.3389/fphar.2020.00332

17. 17. Farmer AD, Wood E, Ruffle JK. An approach to the care of patients with irritable bowel syndrome. CMAJ. 16 de marzo de 2020;192(11):E275-82. https://doi.org/10.1503/cmaj.190716

18. Brugnatelli V, Turco F, Freo U, Zanette G. Irritable Bowel Syndrome: Manipulating the Endocannabinoid System as First-Line Treatment. Front Neurosci. 2020;14:371. https://doi.org/10.3389/fnins.2020.00371 